\title{
Nonlinear experiments : a saturation example
}

\author{
Cristina Lampón* Ramon Costa-Castelló ${ }^{* *}$ \\ Sebastián Dormido*** \\ * Universitat Politècnica de Catalunya (UPC) (e-mail: \\ cristina.lampon@upc.edu \\ ** Universitat Politècnica de Catalunya (UPC) (e-mail: \\ ramon.costa@upc.edu \\ *** Universidad Nacional de Educación a Distancia (UNED) (e-mail: \\ sdormido@dia.uned.es)
}

\begin{abstract}
Most control system are influenced by static nonlinearities. It is important for an engineer to understand which is the effect of these nonlinearities. Unfortunatelly most handson laboratory can not be used for these type of experiences. In this paper it is described an ongoing project which adapts most conventional experimental setups to allow them to be used for nonlinear experiencies. In particular, the saturation case is described.
\end{abstract}

Keywords: static nonlinearities, saturation, phase plane, describing function

\section{INTRODUCTION}

Practical works are an important component of engineering studies and in particular in control engineering. Most practical works in this area are based on simple plants which can be described through linear models or system which have an smooth nonlinear behavior which can be described by a smooth nonlinear model (i.e inverted pendulum, Twin Rotor, tanks)(Bayrakceken and Arisoy, 2013). On the contrary, very few practical works have focused on the effect of static nonlinearities such as saturation, dead-zone or rele(Ortega et al., 2000). This type of discontinuous nonlinearities are present in many control systems but, although they produce very interesting behaviours, few didactic equipment has focus on this type of components. As far as the authors known there's no commercial products which can be used for this purpose.

Although most relevant theory in nonlinear systems is Lyapunov theory(Gordillo, 2009), other methodologies can be of interest in many practical cases. There exist many closed-loop systems which can be described through a linear model plus a static nonlinearity (Lure systems), this type of system can be studied through classical control methods such as phase plane and describing function (Slotine and Li, 1991; Khalil, 2002). These methods although are simple and approximate contribute to extend the tools engineering students can use to analyze and design closedloop system.

Although most systems, including didactic platforms, contain static nonlinearities it is difficult to develop practical experiments based on these nonlinearities because they are not easy to access or isolate for the rest of the system. In order to overcome these problems the authors have development a module which can be connected to didactic plant so it shows nonlinear behaviors. This module does not depend on the concrete plant (i.e. it can be connected to

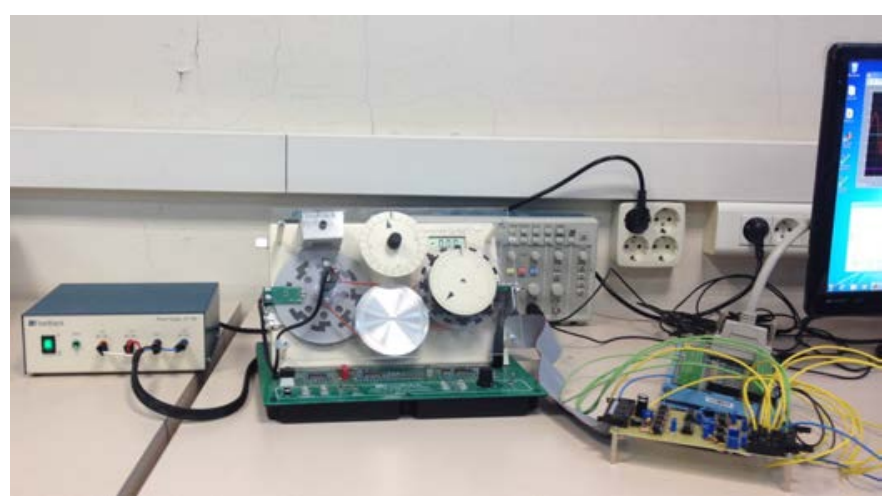

Fig. 1. Plant and NL nonlinear module board. Version 3.

different types of plants), and allows to introduce measuring points at several points so that the analysis is possible. This paper describes some components of the module and show a practical experience which can be developed based on the developed module. In a near future this module will be integrated in a virtual/remote laboratory (CostaCastelló et al., 2010) so it can be used remotely without limitations.

This paper is organized as follows, section 2 contains a description of the module developed to introduce nonlinearities, section 3 shows the behavior a closed-loop system which contains a saturation in it, finally section 4 contains some conclusions and future works.

\section{BOARD}

From many time ago, the automatic control department (ESAII) from UPC has been teaching different aspects in automatic control including nonlinear systems. To support this studies some practical experiments have been designed, between others a module to include nonlinearities 


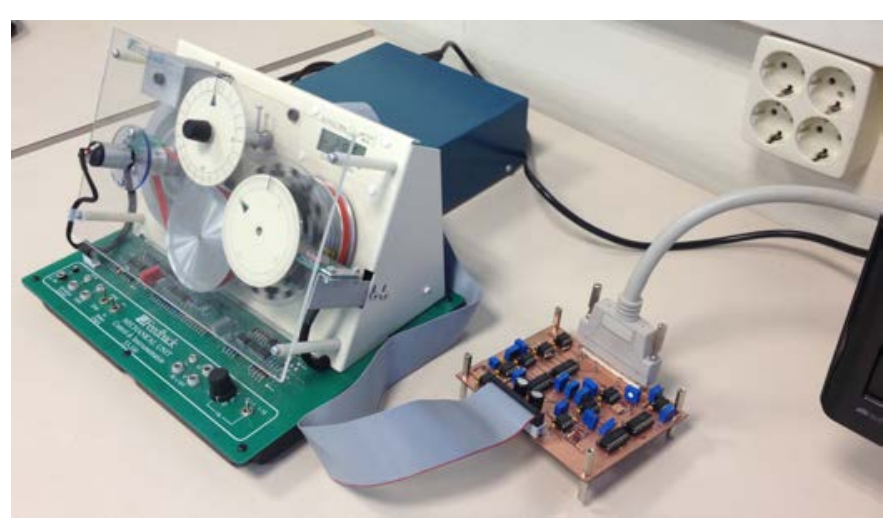

Fig. 2. Plant and NL nonlinear module board. Current status.

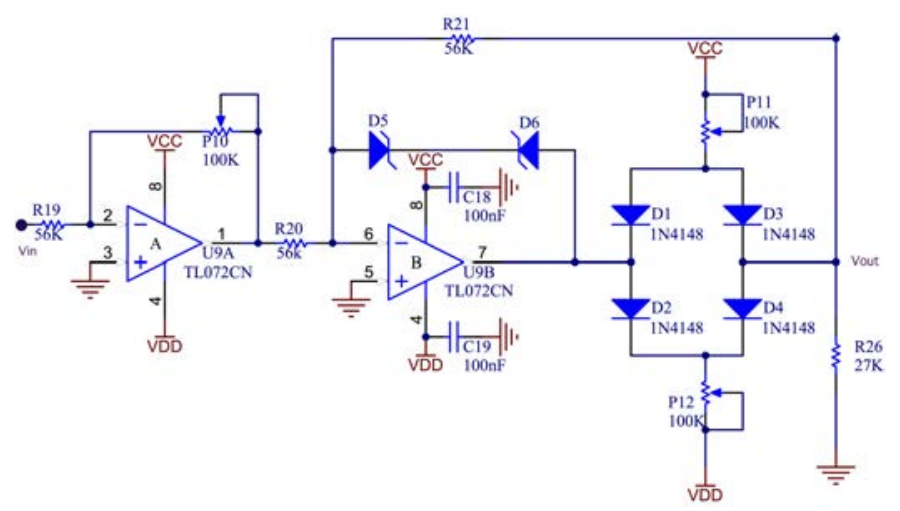

Fig. 3. Saturation implementation schematics.

in a closed-loop system containing a DC motor. It is not the goal of this module to construct a self contained experimental setup, on the contrary it is designed to take profit from an existing device (FEEDBACK Mechanical Unit 33100). This device can be used to develop conventional practical works for introductory courses (i.e. speed control and position control). Figure 1 shows a view of the device an a previous version of the developed board. The complete system is connected to a $\mathrm{PC}$, which contains an $\mathrm{AD} / \mathrm{DA}$ board (Advantech PCI-1711), through an interconnection board (Advantech PCLD-8710). The AD/DA board is used to visualize the evolution of most relevant variables (i.e. speed, position, control action) and to generate signals (i.e. references and identification signals). An environment based on MATLAB/Simulink helps to handle the AD/DA board in a friendly manner.

Figure 2 shows the current version of the nonlinearity module. It is important to see that current status of this module has a simpler and cleaner interconnection; power is taken directly from the mechanical device and the module is directly connected to the AD/DA board. Although the module is optimized for the FEEDBACK Mechanical Unit 33-100 it can be easily used with other components.

Although the board performs different functions, in this work only the saturation functionality will be described. This functionality is implemented using analog electronics, Figure 3 shows the circuit which implements the saturation functionality. The circuit is composed by two parts, the first is an inverter amplifier with a gain which can be changed through a potentiometer; and the second is a

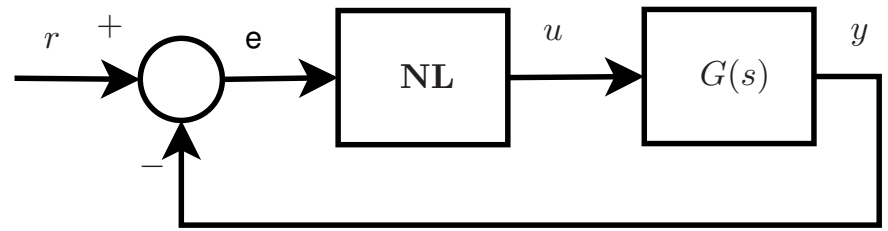

Fig. 4. Studied system block scheme

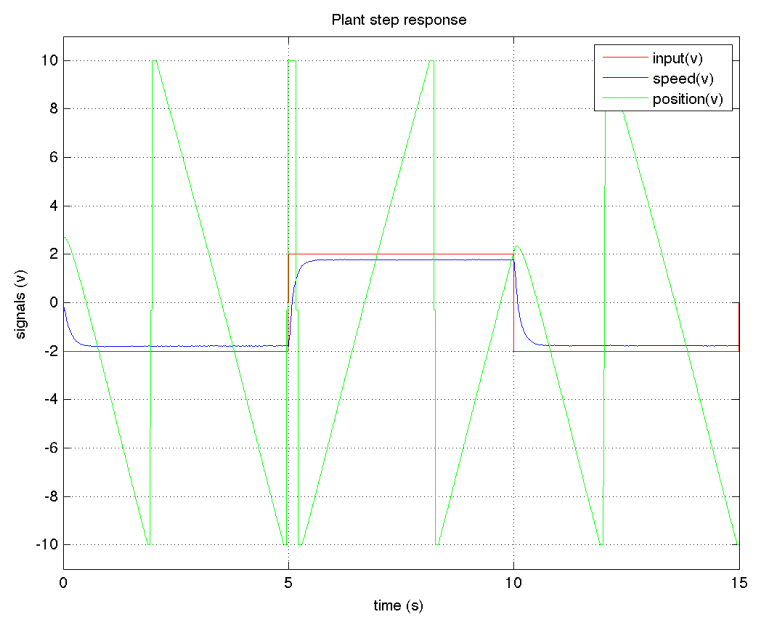

Fig. 5. Plant step response.

limiter which limits can be changed through two potentiometer (one for the upper and another for the lower limits) (Wait et al., 1975).

\section{EXPERIMENTAL RESULTS}

In this section several experiments will be described. These experiments constitute an experimental session performed by the students during a hands-on laboratory. Figure 4 shows the block scheme of the system closed-loop system under study. As it can be seen, it is assumed that the system is composed by a linear system $G(s)$ and a nonlinearity $N L$, which are separated and feedback connected. The linear part corresponds to the DC-motor while the nonlinearity corresponds to the developed board.

Firstly, the linear part it is identified, to do this the closedloop scheme is opened an a step signal is placed in the linear system input, $u$. Figure 5 shows the evolution of the motor speed and position when an square signal is introduced in the input. As it can be shown the speed behaves as a first order system while the output of the position corresponds to an scaled integral of the speed. As the position is measured using a one turn potentiometer it can be shown a discontinuity when the position goes through $\pi$ rad or $-\pi$ rad.

Having this experiment in mind the model for the DCmotor model can be written as:

$$
G(s)=\frac{k}{\tau s+1} \frac{1}{N \cdot s} .
$$

The values of $k$ and $\tau$ can be directly obtained from the experiments while $N=32$ is obtained from the plant structure (reduction). 


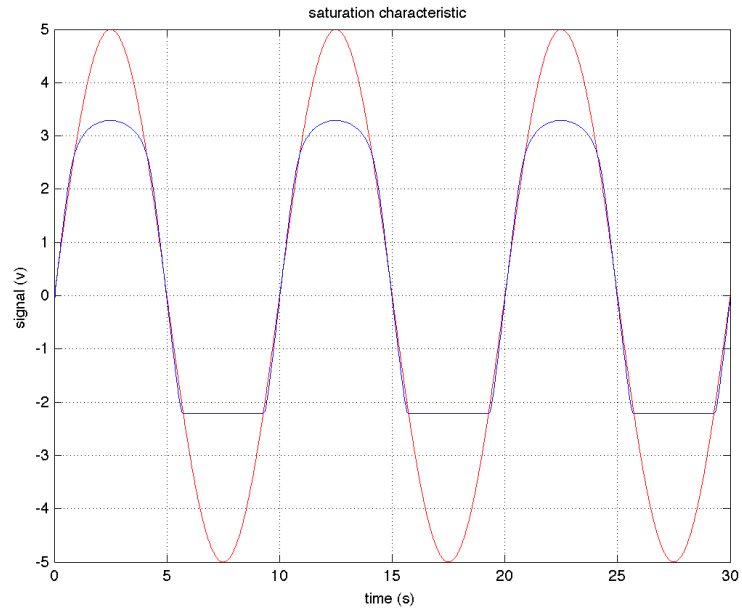

Fig. 6. Saturation input and output signals.

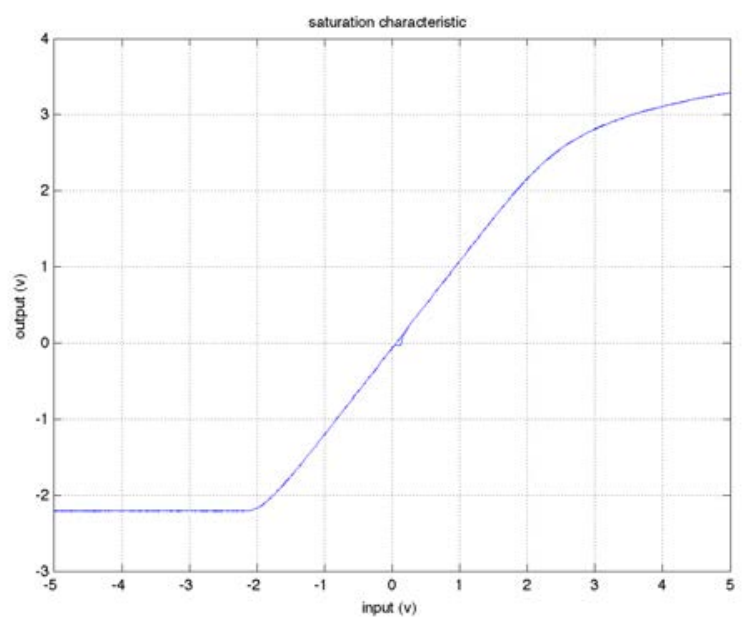

Fig. 7. Saturation input/output relationship.

After that, the nonlinearity will be characterized. To do this a sinusoidal signal is introduced to the nonlinearity input $(e)$ and the output, $u$, is observed (Figure 6). As it can be seen the output is truncated both from the lower and upper parts. Note that the truncation is different in the bottom part than in the upper one, this is due to the limits fixed through the potentiometers. Figure 7 shows the input-output static relationship obtained from this experiment, note that bottom part is very similar to a saturation while the upper part is not so sharp.

A method which can be applied to analyze the behavior of the system in Figure 4 is the describing function. Figure 8 shows the magnitude of the first harmonic component. As it can bee seen it is quite similar to that of an ideal saturation (Slotine and Li, 1991). Although it is not shown in this work, the phase introduced by the function is almost zero, so it can be considered a real describing function.

Additionally, Figure 9 shows the values of the offset value that will appear at the output of the nonlinearity. For small values of the amplitude, $A$, it is very small while it increases for higher values. This could be explained in terms of the asymmetry in the input-output relationship (Figure 7).

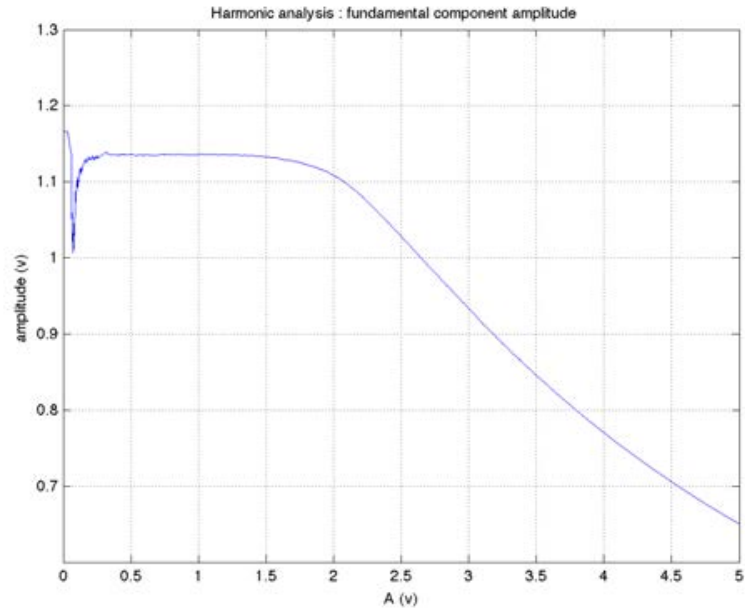

Fig. 8. Nonlinearity Describing Function : Fundamental Component Magnitude.

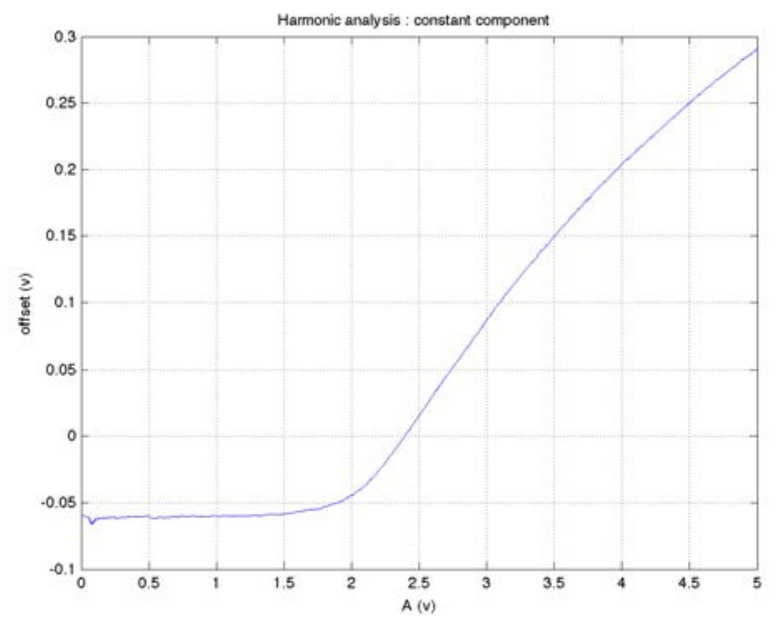

Fig. 9. Nonlinearity Describing Function : Constant Component.

As the describing function is real and the linear system, $G(s)$, is a second order no intersection between the describing function and $G(j \omega)$ will exist in the Nyquist; consequently the closed-loop system with the saturation will be stable if the closed-loop system without the saturation is stable. As it is well-know saturation-like nonlinearities do not introduce limit cycles or unstable behavior. As the describing function method only provides information about the steady-state regime, this does not mean that both closed-loop systems will have the same transient behavior.

Figure 10 shows the step response of the linear position closed-loop, as it can been shown the position perfectly tracks the reference in steady-state (a slight difference between the position and the reference exist due to calibration problems). This behavior corresponds to the behavior of a type one linear system (Guzmán Sánchez et al., 2012). Figure 11, shows the step response of the position closedloop system with the saturation, as it can be shown the position perfectly tracks the reference in steady-state as in the linear case. As predicted by the describing function 


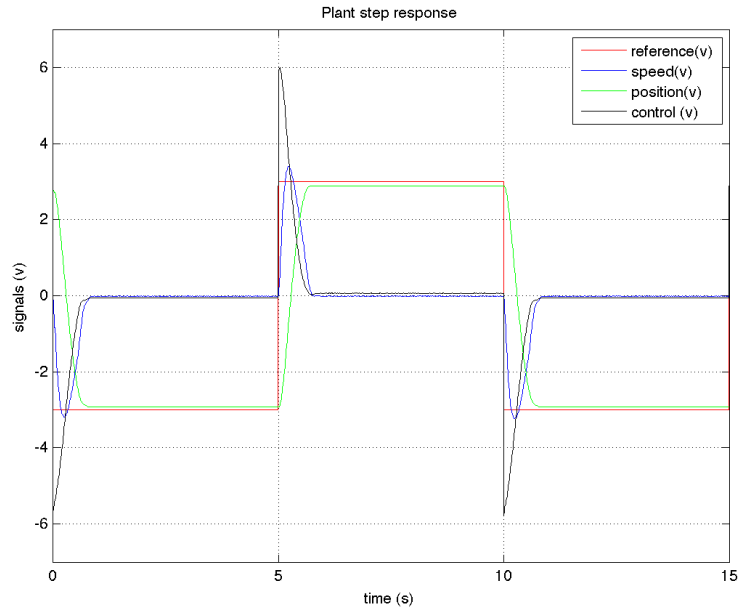

Fig. 10. Closed-loop linear system step response.

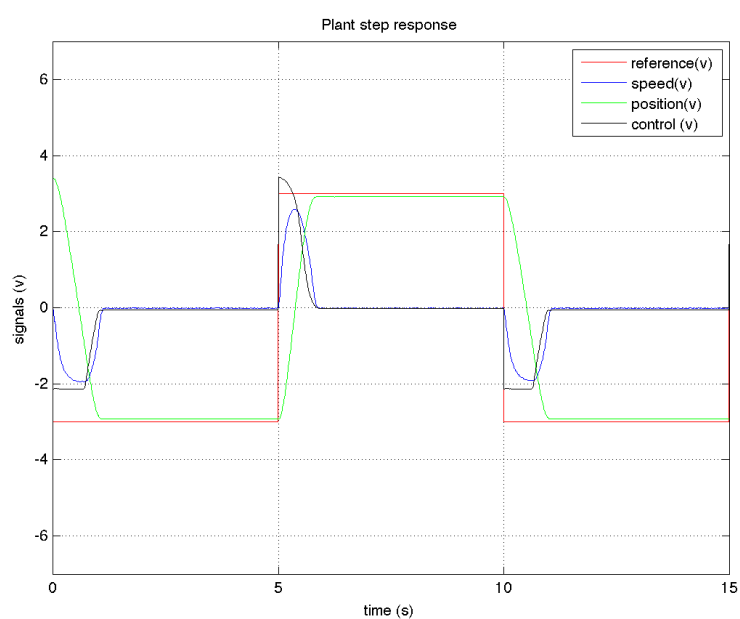

Fig. 11. Closed-loop (with saturation) system step response.

the steady-state behavior is the same for the linear and the nonlinear case. Although the steady-state is the same, the transient behavior is quite different, two main differences exist; the speed takes smaller values than the ones in the linear case and behavior is not symmetric. Both changes can be explained in terms of the nonlinearity characteristics, a between visualization of the differences can be obtained through the phase plane.

Figure 12 shows the closed-loop system linear phase plane. Speed and position variables are selected as state variables. As the closed-loop system is second order, the phase-plane contains the complete system information. As it can be seen the phase plane can be explained in terms of two exponentials curves, one for the positive reference and one for the negative reference. Figure 13 shows the phase plane for the system containing the saturation, the trajectories converge to the same equilibrium points than in the linear case, but trajectories are not the same. Note that the system trajectories are not symmetric, positive speed take higher values than the negative ones.

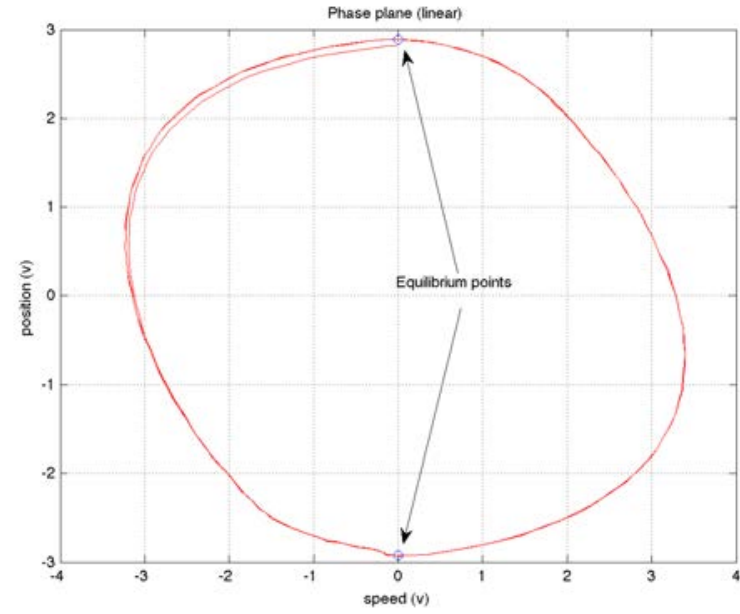

Fig. 12. Closed-loop linear system phase-plane.

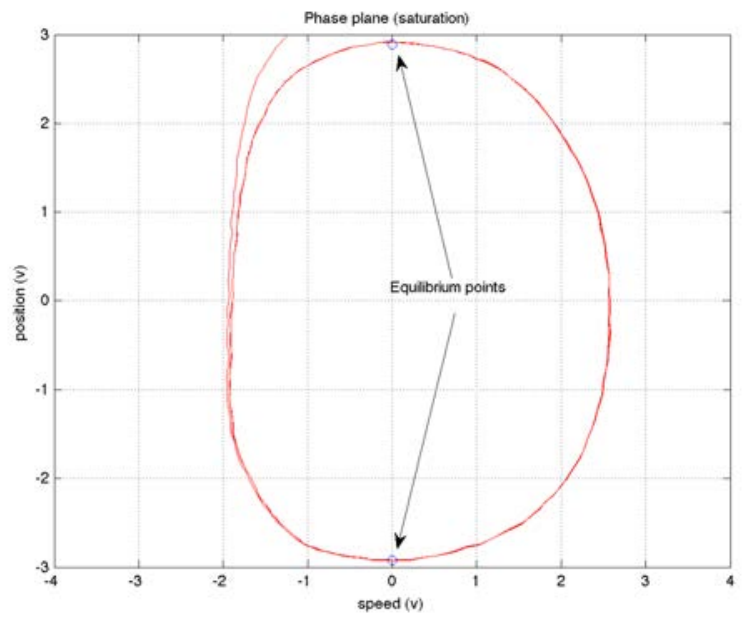

Fig. 13. Closed-loop (with saturation) system phase-plane.

\section{CONCLUSIONS}

In this work a setup developed to do experiments on nonlinear systems has been described. This setup is composed by a commercial DC motor and a developed analog electronics board. This board handles the connection between the setup and a PC with an AD/DA card.

Described setup has been used in practical works on phaseplane and describing function in engineering studies.

Currently, the authors are working to improve the setup in order to include additional nonlinearities, develop a board which allows to change the nonlinearity characteristics, and develop a virtual/remote laboratory which allows to use the setup remotely.

\section{ACKNOWLEDGEMENTS}

This work has been funded by the National Plan Project DPI2012-31303 of the Spanish Ministry of Science and Innovation. 


\section{REFERENCES}

Bayrakceken, M. and Arisoy, A. (2013). An educational setup for nonlinear control systems: Enhancing the motivation and learning in a targeted curriculum by experimental practices [focus on education]. Control Systems, IEEE, 33(2), 64-81. doi:10.1109/MCS.2012.2234971.

Costa-Castelló, R., Vallés, M., Jiménez, L., Diaz-Guerra, L., Valera, A., and Puerto, R. (2010). Integración de dispositivos físicos en un laboratorio remoto de control mediante diferentes plataformas: Labview, matlab y c/c++. Revista Iberoamericana de Automática e Informática Industrial (RIAI), 7(1), 23 - 34. doi: doi:0.1016/S1697-7912(10)70005-4.

Gordillo, F. (2009). Estabilidad de sistemas no lineales basada en la teoría de liapunov. Revista Iberoamericana de Automática e Informática Industrial (RIAI), 6(2), 5
- 16. doi:10.1016/S1697-7912(09)70088-3.

Guzmán Sánchez, J.L., Costa Castelló, R., Berenguel Soria, M., and Dormido Bencomo, S. (2012). Control automático con herramientas interactivas. Pearson. ISBN: 9788483227503.

Khalil, H.K. (2002). Nonlinear Systems. Prentice Hall, Upper Saddle River, New Jersey, 3. edition edition.

Ortega, M., Aracil, J., Gordillo, F., and Rubio, F. (2000). Bifurcation analysis of a feedback system with dead zone and saturation. Control Systems, IEEE, 20(4), 91-101. doi:10.1109/37.856182.

Slotine, J.J.E. and Li, W. (1991). Applied Nonlinear Control. Prentice-Hall Inc. ISBN 0-13-040890-5.

Wait, J.V., Huelsman, L.P., and Korn, G.A. (1975). Introduction to operational amplifier theory and applications. McGraw-Hill Inc. US. ISBN : 0070677654. 